\title{
Correspondence
}

\section{Tilorone: A Broad-Spectrum Antiviral For Emerging Viruses}

\author{
Sean Ekins $1^{*}$ and Peter B. Madrid2
}

1Collaborations Pharmaceuticals, Inc., 840 Main Campus Drive, Lab 3510, Raleigh, NC27606, USA

2SRI International, 333 Ravenswood Avenue, Menlo Park, CA 94025, USA.

*To whom correspondence should be addressed: Sean Ekins, E-mail address: sean@collaborationspharma.com, Phone: +1 215-687-1320

Short running title: Tilorone

Keywords: Antiviral, broad spectrum, emerging viruses 
In recent years we have witnessed major Ebola virus outbreaks in Africa, the Zika outbreak in Brazil and now the novel coronavirus (COVID-19) in China. These viruses are rarely confined to their original locations and thus create challenges in containment. Newer viruses also lack available approved treatments and indicate the need for broader spectrum antivirals. We now highlight one such molecule, tilorone dihydrochloride (tilorone, Amixin ${ }_{\circledast}$ or Lavomax®) which is currently registered for human use in Russia, Ukraine, Kazakhstan, Belarus, Armenia, Georgia, Kyrgyzstan, Moldova, Turkmenistan, and Uzbekistan as an antiviral (influenza, acute respiratory viral infection, viral hepatitis, viral encephalitis, myelitis, and others) and immunomodulating medication. It is also included in the list of essential medicines of the Russian Federation. In vivo efficacy studies dating back to 1970 support possible uses against a broad array of viruses including influenza A, influenza B, herpes simplex virus 1, West Nile virus, Mengo virus, Semliki Forest virus, vesicular stomatitis virus and encephalomyocarditis virus [1-3]. More recently we recently demonstrated $90-100 \%$ survival in mice infected with Ebola then treated with tilorone [4]. These results led us to more broadly profile the antiviral spectrum of activity and we identified activities against Chikungunya virus (CHIK) and Middle Eastern Respiratory Syndrome Coronavirus (MERS-CoV) (Table 1), the latter of which also agrees with others [5]. These combined observations suggest tilorone is a potential broad-spectrum antiviral that may have utility against additional coronaviruses. Tilorone has never been evaluated and tested for safety and efficacy under studies that meet current ICH and FDA guidelines and regulations. Recent virus outbreaks suggest the urgent need for reassessment of this compound as a broad-spectrum antiviral as we have yet to fully appreciate the utility of a drug discovered 50 years ago. 


\section{Acknowledgments}

We kindly acknowledge NIH funding: R21TR001718 from NCATS (PI - Sean Ekins). Dr.

Mindy Davis is gratefully acknowledged for assistance with the NIAID virus screening capabilities, Task Order number B22.

\section{References}

1. Krueger, R.E. and G.D. Mayer, Tilorone hydrochloride: an orally active antiviral agent. Science, 1970. 169(3951): p. 1213-4.

2. Vargin, V.V., W. Zschiesche, and B.F. Semenov, Effects of tilorone hydrochloride on experimental flavivirus infections in mice. Acta Virol, 1977. 21(2): p. 114-8.

3. Kuehne, R.W., W.L. Pannier, and E.L. Stephen, Evaluation of various analogues of tilorone hydrochloride against Venezuelan equine encephalitis virus in mice. Antimicrob Agents Chemother, 1977. 11(1): p. 92-7.

4. Ekins, S., et al., Efficacy of Tilorone Dihydrochloride against Ebola Virus Infection. Antimicrob Agents Chemother, 2018. 62(2).

5. Shen, L., et al., High-Throughput Screening and Identification of Potent BroadSpectrum Inhibitors of Coronaviruses. J Virol, 2019. 93(12). 


\begin{tabular}{llllllc}
\hline \multicolumn{1}{c}{ Virus } & \multicolumn{1}{c}{ Strain } & \multicolumn{1}{c}{ Genus } & Type & Cell line & $\begin{array}{c}\mathrm{EC}_{50} \\
(\mu \mathrm{M})\end{array}$ & $\begin{array}{c}\mathbf{C C}_{50} \\
(\mu \mathrm{M})\end{array}$ \\
\hline CHIKV & S27 (VR-67) & Alphavirus & + ssRNA & Vero 76 & $4.2^{*}$ & 32 \\
\hline MERS-CoV & EMC & Betacoronavirus & + ssRNA & Vero 76 & $3.7^{*}$ & 36 \\
\hline
\end{tabular}

Table 1. Antiviral screening data for Tilorone generated under the NIAID-DMID NCEA antiviral in vitro screening services.

* In vitro antiviral data in Vero 76 cells may underestimate antiviral activity due to lacking IFN pathways. 